\title{
Protocolo de uso de fios ortodônticos em aparelhos autoligáveis
}

\author{
Protocol of the use of orthodontic wires in self-ligating appliances \\ Protocolo de uso de alambres de ortodoncia en aparatos de autoligado
}

Recebido: 19/03/2021 | Revisado: 26/03/2021 | Aceito: 09/04/2021 | Publicado: 18/04/2021

Laylla Carneiro da Silva Haas

ORCID: https://orcid.org/0000-0002-5866-4219

Faculdade FAIPE, Brasil

Faculdade Garça Branca Pantanal, Brasil

E-mail: layllacarneiro@hotmail.com

Luiz Eduardo Alessio Junior

ORCID: https://orcid.org/0000-0001-6226-3787 Faculdade FAIPE, Brasil

Faculdade Garça Branca Pantanal, Brasil

E-mail: lui.alessio@gmail.com

Melissa Guimarães Crepaldi

ORCID: https://orcid.org/0000-0002-2664-1426

Faculdade FAIPE, Brasil

Faculdade Garça Branca Pantanal, Brasil

E-mail: melissagcrepaldi@hotmail.com

Viviane Curi

ORCID: https://orcid.org/0000-0002-7931-3186 Faculdade FAIPE, Brasil

Faculdade Garça Branca Pantanal, Brasil

E-mail: curiviviane@gmail.com

Jaime Rodolfo Gamarra Suárez

ORCID: https://orcid.org/0000-0002-4426-2083

Faculdade FAIPE, Brasil

Faculdade Garça Branca Pantanal, Brasil

E-mail: clinicaortodoncica@hotmail.com

Kelly Maria Silva Moreira

ORCID: https://orcid.org/0000-0002-1137-3908

Faculdade FAIPE, Brasil

Faculdade Garça Branca Pantanal, Brasil

E-mail: kellynhaodonto@yahoo.com.br

Priscila Vieira da Silva

ORCID: https://orcid.org/0000-0002-6295-2344

Faculdade FAIPE, Brasil

Faculdade Garça Branca Pantanal, Brasil

E-mail: privieira.odonto@gmail.com

Marcus Vinicius Crepaldi

ORCID: https://orcid.org/0000-0001-7665-0585 Faculdade FAIPE, Brasil

Faculdade Garça Branca Pantanal, Brasil

E-mail: marcuscrepaldi@yahoo.com.br

\section{Resumo}

Devido à grande variedade de fabricantes de aparelhos ortodônticos autoligados, dúvidas em relação ao protocolo de utilização e sequência dos fios nestes aparelhos podem surgir. Desta forma o conhecimento das propriedades mecânicas dos fios ortodônticos, facilita a escolha mais adequada para cada fase dos tratamentos. A evolução tecnológica de manufatura dos fios e a elaboração de novas técnicas ortodônticas levam a uma procura por uma melhor qualidade das ligas, a fim de torná-los biologicamente mais efetivos no que diz respeito aos dentes e tecidos suporte. Este trabalho apresenta o protocolo de fios para cada fases de tratamento; o protocolo de fios recomendado pelos diferentes fabricantes e o protocolo do fio Bioforce utilizada na clínica FAIPE.

Palavras-chave: Ortodontia; Desenho de aparelho ortodôntico; Aparelhos ortodônticos fixos.

\begin{abstract}
Due to the wide variety of manufacturers of self-ligating orthodontic appliances, doubts regarding the protocol of use and the sequence of the archwires in these appliances may arise. In this way, the knowledge of the mechanical properties of orthodontic wires facilitates the most appropriate choice for each phase of treatments. The technological evolution of the manufacture of the wires and the development of new orthodontic techniques lead to a search for a better quality of the alloys, in order to make them biologically more effective with regard to the teeth and supporting
\end{abstract}


tissues. This work presents the wire protocol for each treatment phase; the wire protocol recommended by different manufacturers and the Bioforce archwire protocol used at the FAIPE clinic.

Keywords: Orthodontics; Orthodontic appliance design; Orthodontic fixed appliances.

\section{Resumen}

Debido a la gran variedad de fabricantes de aparatos de ortodoncia de autoligado, pueden surgir dudas sobre el protocolo de uso y la secuencia de los arcos en estos aparatos. De esta forma, el conocimiento de las propiedades mecánicas de los alambres de ortodoncia, facilita la elección más adecuada para cada fase de los tratamientos. La evolución tecnológica de la fabricación de los alambres y el desarrollo de nuevas técnicas de ortodoncia conducen a la búsqueda de una mejor calidad de las aleaciones, con el fin de hacerlas biológicamente más efectivas con respecto a los dientes y tejidos de soporte. Este trabajo presenta el protocolo de alambre para cada fase de tratamiento; el protocolo de alambre recomendado por diferentes fabricantes y el protocolo de arco Bioforce utilizado en la clínica FAIPE.

Palabras clave: Ortodoncia; Diseño de aparato ortodóncico; Aparatos Ortodóncicos fijos.

\section{Introdução}

Desde a antiguidade, dentes apinhados e irregulares tem sido um problema para alguns indivíduos e tentativas para corrigir essas desordens datam de pelo menos, 1.000 anos a.C. (Vilella et al., 1995).

A Ortodontia é a mais antiga das especialidades da Odontologia, tendo sido a primeira a se organizar de fato e de direito. Angle em 1915 desenvolveu o arco de cinta (ribbon arch appliance). Braquetes foram introduzidos pela primeira vez nesse novo aparelho, sendo caracterizados por possuírem o slot numa posição vertical. O braquete do arco de canto (Edgewise appliance) foi apresentado em 1928/29, e consistia de uma caixa retangular com uma fenda no meio (slot), cujas dimensões eram de 0,022 " x 0,028". Esse desenho proporcionava maior precisão e um mecanismo de torque muito mais eficiente. A partir dos anos 60, começou a surgir nos Estados Unidos a ideia de se introduzir inclinações nos slots ou nas bases dos braquetes, denominou essa técnica como Straight-wire, ou arco reto (Vilella et al., 1995).

Introduzidos no início do século 20, o conceito de braquetes sem ligaduras surgiu com o aparelho Russell Lock, que foi uma tentativa de alcançar eficiência clínica associada à redução do tempo gasto com a ligação dos braquetes (Sathler et al., 2011). O pressuposto desse conceito é que o controle adicional dado pelo fechamento do braquete provoca um assentamento total do fio dentro do slot, propiciando melhor controle tridimensional na posição dos dentes (Ursi \& Almeida, 2010).

A classificação mais tradicional dos braquetes autoligáveis divide esses acessórios em três tipos, de acordo com o grau de pressão do sistema aplicado ao fio. Eles podem ser ativos, quando o sistema pressiona o fio dentro da canaleta; passivos, quando o sistema permite liberdade do fio na canaleta; ou interativos, quando os braquetes autoligáveis exercem pressão em fios mais espessos, mas permitem liberdade de fios menos calibrosos (Sathler et al., 2011).

O conhecimento das propriedades e características dos fios ortodônticos permite que o profissional realize a movimentação ortodôntica de forma mais eficiente, evitando danos aos tecidos de suporte e aos dentes. A mecânica ortodôntica baseia-se no princípio de acúmulo de energia elástica e então transformação da mesma em mecânica, por meio da movimentação dentária. Os ajustes dos aparelhos ortodônticos promovem o armazenamento e controle do mecanismo de transferência e da distribuição de forças. Para um excelente controle da movimentação dentária, é necessária a aplicação de um sistema de forças ideal, que deve ser devidamente guiado por meio de acessórios, tais como os fios ortodônticos (Quintão \& Brunharo, 2009).

O movimento ortodôntico envolve a interação adequada de fatores relacionados ao paciente, à mecânica aplicada, aos dentes e suas estruturas de suporte. Particularmente é dependente da ação dos fios ortodônticos, conforme suas características estruturais e mecânicas. Por muito tempo os fios de aço inoxidável predominaram na Ortodontia, mas o advento de novas ligas metálicas tornou diversificado o universo de fios disponíveis. Estas novas ligas têm propiciado algumas alterações no 
protocolo de tratamento, encurtando o tempo de cadeira, bem como do tratamento como um todo (Gurgel, Ramos \& Kerr, 2001).

O objetivo deste trabalho é descrever em cada fase específica do tratamento qual tipo de fio deve ser utilizado, a sequência de fios recomendada pelos diferentes fabricantes e o protocolo do fio Bioforce utilizada na clínica FAIPE.

\section{Metodologia}

O presente estudo é uma revisão integrativa da literatura, executada por meio do procedimento de pesquisa bibliográfica de artigos científicos nas bases de dados eletrônicos (Pereira et al., 2018). Além disso, foi apresentado o protocolo de uso de fios ortodônticos para aparelhos fixos autoligados, sugerido pela faculdade Faipe.

\section{Revisão de Literatura}

A especialidade mais antiga a se organizar foi a Ortodontia. Dentes apinhados e irregulares tem sido um problema e tentativas para corrigir essas alterações datam de pelo menos, 1.000 anos a.C. encontrados em escavações gregas e etruscas. (Motta et al., 2003; Vilella, 2007).

Pierre Fauchard (1678-1761) apresentou um aparelho denominado bandeau, que consistia de uma tira de metal flexionada em forma de arco e perfurada em locais adequados. Foi o primeiro arco expansor introduzido na Ortodontia. Naquela época, a principal finalidade do tratamento era o alinhamento dos dentes anteriores, principalmente os da maxila. Os métodos empregados por Fauchard incluíam luxação e "reposicionamento" de dentes mal posicionados (Vilella, 2007).

Em Baltimore College of Dental Surgery (Maryland) no dia 06 de março de 1840, fundou-se a primeira Escola de Odontologia no mundo (Vilella, 2007). Harris já preconizava conceitos básicos da Ortodontia atual, tais como a extração de dentes permanentes para a correção dos apinhamentos dentários severos, como também a necessidade de remoção da causa das irregularidades, a fim de que a estabilidade do tratamento pudesse ser alcançada (Vilella, 2007).

No final do século XIX os ortodontistas já podiam contar com fio, bandas, cimento e solda. Angle, então, projetou uma aparelhagem padrão, composta por uma coleção de peças pré-fabricadas (Angle System), que podiam ser montadas em várias combinações. Sua classificação para as más oclusões, publicada em 1899, continua sendo a mais utilizada e amplamente aceita até os dias de hoje (Vilella, 2007).

Em 1915, ele desenvolveu o arco de cinta (ribbon arch appliance), muito mais fácil de ser construído e ativado. Braquetes foram introduzidos pela primeira vez nesse novo aparelho, sendo caracterizados por possuírem o slot numa posição vertical. O braquete do arco de canto (Edgewise appliance) foi apresentado em 1928/29, e consistia de uma caixa retangular com uma fenda no meio (slot), cujas dimensões eram de 0,022” x 0,028”. Esse desenho proporcionava maior precisão e um mecanismo de torque muito mais eficiente (Vilella, 2007).

A partir dos anos 60, começou a surgir nos Estados Unidos a ideia de se introduzir inclinações nos slots ou nas bases dos braquetes. Lawrence F. Andrews denominou essa técnica como Straight Wire, ou arco reto. Também em 1976, Robert Murray Ricketts apresentou a técnica Bioprogressiva, considerada pelo autor uma evolução da técnica Edgewise (Vilella,1995).

A partir de meados da década de 1990 tornaram-se viáveis os braquetes autoligados (Speed system, Time bracket, Damon SL e Twinlock). Esses braquetes apresentam uma superfície de metal embutida que pode ser aberta e fechada, dispensando ligaduras elásticas (Vilella, 2007).

Os bráquetes autoligados, ainda que não seja uma unanimidade entre todos os autores que compõem a teoria escrita até o momento, vem se tornando fortes aliados dos ortodontistas nos tratamentos relacionados a má formação dos dentes.

Calcado no oferecimento de vantagens sobre os dispositivos convencionais, através de uma técnica que promete menos atrito 
no sistema, menos tempo de tratamento, menos visitas ao dentista, menor desconforto, entre outros benefícios, este sistema viu sua notoriedade ser alavancada entre os profissionais da área de odontologia, que buscam sempre melhores resultados para seus pacientes (Zanelato, 2016).

Em 1887, Edward Angle utilizou ligas de níquel e prata para confecção de acessórios ortodônticos. Posteriormente, ele as substituiu por ligas de cobre, zinco e níquel, sem adição de prata. E por fim, as ligas de ouro surgiram e se tornaram as de escolha. Até a década de 1930, a liga de ouro foi a mais utilizada na fabricação dos acessórios ortodônticos (Quintão \& Brunharo, 2009).

Em 1933, Archie Brusse, fundador da empresa denominada Rocky Mountain, sugeriu pela primeira vez a utilização clínica do aço inoxidável na Ortodontia. Na década de 1960, foram introduzidas as ligas de cobalto e cromo na Ortodontia. As primeiras aplicações clínicas da liga beta-titânio na Ortodontia ocorreram na década de 1980, quando foi sugerida uma forma de titânio denominada "de alta temperatura". Desde então, essas ligas apresentaram ampla aceitação clínica e grande popularidade, e são comercialmente disponibilizadas como "TMA" (titanium molybdenum alloy) (Quintão \& Brunharo, 2009).

No ano de 1972, a Unitek Corporation começou a produção da liga de níquel-titânio para uso clínico, com nome comercial de $\mathrm{Nitinol}^{\circledR}$, composta por 55\% níquel e 45\% titânio, em estrutura equiatômica. As ligas termodinâmicas de níqueltitânio surgiram na década de 90, para fins comerciais. Os fios termodinâmicos de níquel-titânio possuem, além das propriedades de resiliência e recuperação elástica dos fios superelásticos, a característica de se tornarem ativos pelo aumento da temperatura bucal. Sendo assim, os fios de níquel-titânio gradualmente termodinâmicos surgiram no mercado, devido ao consenso de que a resposta dentária à força aplicada e à quantidade de movimentação dentária obtida dependem da área de superfície do periodonto. Desta forma, um arco ideal deve gerar forças suaves e constantes, e também ser capaz de promover variação do nível de força, dependendo da região do periodonto envolvida (Quintão \& Brunharo, 2009).

Existem no mercado 4 grupos de ligas básicas: aço inoxidável; ligas de níquel-titânio (NiTi) com variações durante a fabricação (superelásticos, termodinâmicos e com adição de cobre); ligas de beta-titânio e ligas estéticas de compósitos (Quintão \& Brunharo, 2009).

Os fios ortodônticos de nivelamento e alinhamento iniciais devem ser capazes de gerar forças leves e contínuas e para isso precisam ser flexíveis, e para este propósito foi sugerido uma diversidade de fios de níquel-titânio superelásticos (NiTi) que oferecem uma curva força-flexão com um patamar definido e uma maior faixa de ativação (Quintão \& Brunharo, 2009).

Os fios termoativados caracterizam-se pela distribuição de forças leves e fisiológicas, e possuem temperaturas diferentes para sua ativação 27, 35 e $40^{\circ}$ sendo indicados para diversas fases da mecanoterapia ativa (Rajan et al., 2017). Os fios de Niti com cobre adicionado em sua composição, devido suas propriedades superelásticas, possibilita uma distribuição mais uniforme de força, propiciando a criação de forças leves e contínuas (Macena et al., 2015).

O fio ortodôntico é o principal responsável pela carga que movimenta os dentes, sendo que essa carga deve apresentar-se leve e contínua. Quanto maior o módulo de elasticidade de um fio, maior a sua relação carga/deflexão e maior a sua rigidez, deve-se conhecer, primeiramente, a relação carga/ deflexão e, só então, escolher o fio mais indicado para cada fase da terapia ortodôntica.

O estudo de Schemann-Miguel em 2012, mediante uma amostra de arcos ortodônticos pré-contornados de espessura $0,019 \times 0,025$ " de NiTi convencionais e termoativados, de diversas marcas comerciais do mercado nacional, avaliou a relação carga/deflexão dos fios NiTi convencionais e NiTi termoativados. Foram selecionados 5 arcos pré-contornados de níqueltitânio (NiTi) convencionais e 5 de NiTi termoativados, de quatro marcas comerciais (Abzil, Morelli, 3M Unitek e Ormco), totalizando 40 arcos. Esses arcos foram instalados em manequins ortodônticos com ausência do elemento 11, para que o fio pudesse ser testado nesse segmento com o auxílio de uma máquina universal de ensaios acoplada a um computador. Comparados os valores médios de carga/deflexão dos fios de NiTi convencionais, observou-se que os que apresentaram a 
menor relação carga e deflexão foram os da 3M Unitek, seguidos pela Ormco, Morelli e Abzil. Nos termoativados, observouse que os que apresentaram a menor relação carga/deflexão foram os da Ormco, demonstrando valores inferiores aos seguidos pela 3M Unitek, Abzil e Morelli. Analisando-se comparativamente os valores médios da relação carga/deflexão nos dois tipos de fios, observou- se que os termoativados apresentaram valores médios da relação carga/deflexão inferiores, mantendo essa tendência durante todo o ensaio. No entanto, notou-se que na deflexão de $2 \mathrm{~mm}$, os fios termoativados da marca Morelli e os convencionais 3M Unitek apresentaram valores médios da relação carga/deflexão muito próximos e, estatisticamente, não significativos. Classificando-os de acordo com a relação carga/deflexão, dos fios mais flexíveis para os mais rígidos, na seguinte ordem: fios Ormco termoativados, 3M Unitek termoativados, Abzil termoativados, Morelli termoativados, 3MUnitek convencional, Ormco convencional e Abzil e Morelli convencionais (Schemann-Miguel et al., 2012).

O uso dos fios ortodônticos de Níquel-Titânio (Ni-Ti) termoativados se popularizou a partir da década de 90 . A memória de forma resultante do efeito pseudoelástico (superelasticidade) e do efeito termoelástico é a característica principal destes fios, e está intrinsecamente relacionada ao Intervalo de Transição Térmica (ITT), onde ocorre a transformação martensítica responsável por estas notáveis propriedades. A temperatura austenítica final (Af) refere-se à temperatura em que o fio atinge a sua fase mais rígida. Sabendo que a temperatura bucal está em torno de $37^{\circ} \mathrm{C}$, o fio ortodôntico termoativado ideal é aquele que apresenta Af igual, ligeiramente abaixo, ou acima da temperatura bucal.

A pesquisa de Spini et al. (2012) avaliou o intervalo de transição térmica de quatro marcas comerciais de fios de Níquel-Titânio termoativados (Aditek, Morelli, Orthometric e Orthosource). Para este estudo, foram selecionadas quatro marcas comerciais de fios de Ni-Ti termoativados de diâmetro 0,019" x 0,025”: Nitinol Termoativado (Aditek, Cravinhos, Brasil); Thermo Plus (Morelli, Sorocaba, Brasil); Flexy Thermal $35^{\circ} \mathrm{C}$ (Orthometric, Marília, Brasil) e Superthermal Nickel Titanium Arches (Orthosource, Matão, Brasil). As amostras foram obtidas pela parte mais reta de cada arco, seguida levado à máquina de DSC - Differential Scanning Calorimetry (DSC Q20 - TA Instruments). Os arcos testados Nitinol termoativado (Aditek), Thermo Plus (Morelli) e Superthermal Nickel-Titanium Arches (Orthosource) apresentaram temperaturas austeníticas finais abaixo da temperatura ambiente e bucal, estando totalmente austeníticos nestas temperaturas, tornando-lhes de difícil inserção nas canaletas em fases inicias do tratamento ortodôntico. $\mathrm{O}$ arco Flexy Thermal $35^{\circ} \mathrm{C}$ (Orthometric) apresentou temperatura austenítica final acima da temperatura bucal, com grande quantidade de martensita tanto na temperatura ambiente como na temperatura bucal. Neste caso ortodontista deve orientar o paciente a ingerir líquidos quentes para temporariamente aumentar a rigidez do fio e, consequentemente, a força liberada para estimular o movimento dentário. Concluiu-se que o NeoSentalloy F200 (GAC) e o Nitinol Termoativado (Aditek) foram as marcas que demonstraram menores valores de forças nas desativações para deflexões de $3 \mathrm{~mm}$ na temperatura de $37^{\circ} \mathrm{C}$, revelam desta forma superelasticidade que estes arcos apresentam mediante grandes deflexões.

- O ITT do fio Thermo Plus (Morelli) está entre $-64,14^{\circ} \mathrm{C}$ (Mf) e $20,39^{\circ} \mathrm{C}$ (Af), não apresentando memória de forma na temperatura bucal. Este fio não apresenta a propriedade de superelasticidade em aplicações clínicas.

- O ITT do fio Nitinol Termoativado (Aditek) está entre $-42,36^{\circ} \mathrm{C}$ (Mf) e $24,75^{\circ} \mathrm{C}$ (Af), não apresentando memória de forma na temperatura bucal. Este fio não apresenta a propriedade de superelasticidade em aplicações clínicas.

- O ITT do fio Flexy Thermal $35^{\circ} \mathrm{C}$ (Orthometric) está entre $16,68^{\circ} \mathrm{C}$ (Mf) e $45,42^{\circ} \mathrm{C}$ (Af). A temperatura austenítica final para este fio difere em $10,42^{\circ} \mathrm{C}$ do informado pelo fabricante. Este fio apresenta a transição de fases na temperatura bucal e a propriedade de superelasticidade em aplicações clínicas.

- O ITT do fio Superthermal Nickel Titanium (Orthosource) está entre $-68,68^{\circ} \mathrm{C}$ (Mf) e $24,18^{\circ} \mathrm{C}$ (Af), não apresentando memória de forma na temperatura bucal. Este fio não apresenta a propriedade de superelasticidade em aplicações clínicas (Spini et al., 2012). 
$\mathrm{Na}$ fase de alinhamento e nivelamento, os fios de aço inoxidável requerem incorporação de dobras para aumentar a quantidade de fio no espaço entre os braquetes, distribuindo melhor as forças e para compensar a baixa resiliência, quando comparado às outras ligas metálicas. Os fios de aço inoxidável oferecem ótima resistência à corrosão e apresentam maior módulo de elasticidade e limite elástico, tornando-os mais vantajosos do que outras ligas, principalmente quando fios mais rígidos são necessários, como para fechamento de espaços e na finalização ortodôntica (Quintão \& Brunharo, 2009).

Ideais para a fase de nivelamento e alinhamento, as ligas de NiTi apresentam as propriedades: alto limite elástico, baixo módulo de elasticidade e a alta resiliência, tornando-as interessantes para esta fase ortodôntica (Quintão \& Brunharo, 2009). Já os fios de CuNiTi, por serem confeccionados em 3 temperaturas de transição $\left(27^{\circ} \mathrm{C}, 35^{\circ} \mathrm{C}, 40^{\circ} \mathrm{C}\right)$, podem ser utilizados para diferentes objetivos de tratamento. Com a introdução destas ligas no mercado, surgiram alguns protocolos de tratamento ortodôntico biologicamente mais compatíveis devido à liberação de força fisiológicas mais leves, levando a um menor tempo de tratamento (Quintão \& Brunharo, 2009).

\section{Protocolo de Utilização dos Fios para cada Fase do Tratamento}

Através da leitura de vários artigos científicos, da prescrição recomendada pelos fabricantes de fios via e-mail, o protocolo de uso sugerido foi semelhante entre elas chegando a um resultado abaixo:

\section{$1^{\text {a }}$ Fase - Alinhamento e nivelamento (Passiva):}

- Fios: Fios redondos leves de NiTi ou Cooper Niti, variando a espessura de .012", .014" ou .016"

- Objetivo: Alinhamento, nivelamento com correção de rotações, início da forma do arco e prepara para o próximo fio.

- Duração: Essa fase dura em média 6 meses, com intervalos de troca variando de 2 a 3 meses.

\section{$2^{\text {a }}$ Fase - Assentamento dos torques (Ativa):}

- Fios: Fios retangulares NiTi Thermo Ativados (Cooper NiTi) ou Superelásticos .016” x .022”, .017” x. 025” ou $.019 "$ " x $.025 "$ ".

- Objetivo: Início da leitura e controle de torque, controle de espaço, correção total das rotações

- Duração: Esta fase dura em média 10 a 20 semanas.

$3^{\text {a }}$ Fase - Fechamento de espaços (movimentos mesiodistais):

- Fios: Fios retangulares de $\mathrm{CrNi}$ (Aço) nas espessuras .018" x $.025 "$ ou $.019 "$ x $025 "$ ".

- Objetivo: Controle de torque obtido, Controle de torque final, fechamento de espaço posterior, arcos coordenados específicos para o paciente.

- Duração: Esta fase dura em média 20 a 40 semanas.

$4^{\text {a }}$ Fase - Finalização e intercuspidação:

- Fios: arcos de CrNi (Aço) .017" x .025", .018" x .025" ou .019"x.025", fio TMA ou 0,18 aço redondo.

- Objetivos: intercuspidação, correção de linha média, detalhamento final.

- Tempo: 10 a 20 semanas.

\section{Protocolo de utilização dos fios segundo orientação dos fabricantes}

O protocolo de uso segundo os fabricantes como informado via e-mail, através do site da marca ou por portifólio informativo do fabricante, está relatado abaixo no Quadro 1. 
Quadro 1. Protocolo de uso dos fios segundo orientações dos fabricantes.

\begin{tabular}{|c|c|c|c|c|}
\hline & MORELLI & DAMON & ORTHOMETRIC & CLARITY \\
\hline $1^{\text {a }}$ Fase & $\begin{array}{l}\text { Thermo Plus 0.012" } \\
\text { Thermo Plus 0.014" } \\
\text { Thermo Plus 0.016" }\end{array}$ & $\begin{array}{c}\text { 0.014" Damon Cu Niti } \\
\text { ou } 0.016 " \text { Niti }\end{array}$ & $\begin{array}{l}\text { Flex Niti Cooper 0.014" } \\
\text { Flex Niti Cooper 0.018" }\end{array}$ & $\begin{array}{l}0.012 \text { " Super Elástico } \\
0.014 \text { ” Super Elástico }\end{array}$ \\
\hline $2^{\mathrm{a}}$ Fase & $\begin{array}{l}\text { Thermo Plus } \\
0.016 \text { "x0.022" } \\
\text { Thermo Plus } \\
0.017 \text { "x0.025" } \\
\text { Thermo Plus } \\
0.019 \text { "x0.025" }\end{array}$ & $\begin{array}{c}\text { 0.014”x0.025" Damon Cu } \\
\text { Ni-Ti } \\
0.018 \text { "x0.025" Damon Cu } \\
\text { Ni-Ti } \\
0.017 \text { "x0.025" Damon } \\
\text { Ni-Ti } \\
0.019 \text { "x0.025" Damon } \\
\text { Ni-Ti }\end{array}$ & $\begin{array}{l}\text { Flex Niti Cooper } \\
0.014 ” x 0.025 " \\
\text { Flex Niti Cooper } \\
0.017 ” x 0.025 " \\
\text { SuperElastic } \\
\text { 0.019”x 0.025" }\end{array}$ & $\begin{array}{l}\text { 0.014" Nitinol Classic } \\
\text { Tadem } \\
\text { 0.016" Nitinol Classic } \\
\text { Tadem }\end{array}$ \\
\hline $3^{\text {a Fase }}$ & $\begin{array}{l}\text { CrNi (Aço) } 0.018 " \mathrm{x} \\
0.025^{\prime \prime} \\
\text { CrNi (Aço) } 0.019 " \mathrm{x} \\
0.025 "\end{array}$ & $\begin{array}{l}0.019 \text { "x } 0.025 \text { "Aço } \\
0.016 " \text { x } 0.025 \text { "Aço }\end{array}$ & Aço 0.019"x0.025" & $\begin{array}{c}\text { 0.016"x0.025” Beta } \\
\text { Titanium } \\
\text { 0.019”x0.025” Beta III } \\
\text { Titanium ou Aço }\end{array}$ \\
\hline $4^{a}$ Fase & $\begin{array}{l}\text { CrNi (Aço) } 0.017 " \mathrm{x} \\
0.025^{\prime \prime} \\
\text { CrNi (Aço) } 0.018 " \mathrm{x} \\
0.025 " \\
\text { CrNi (Aço) } 0.019 " \mathrm{x} \\
0.025^{\prime \prime}\end{array}$ & $\begin{array}{c}0.019 " \times 0.025 " \mathrm{CrNi} \\
\text { (Aço) }\end{array}$ & $\begin{array}{c}\text { Aço } 0.019 ” \times 0.025 " \\
\text { Braided } 0.019 ” \times 0.025 "\end{array}$ & $\begin{array}{c}0.019 \text { "x } 0.025 \text { " Beta III } \\
\text { Titanium e elásticos de } \\
\text { finalização }\end{array}$ \\
\hline
\end{tabular}

Fonte: Autores.

\section{Protocolo de utilização do fio Bioforce pela clínica FAIPE}

$\mathrm{Na}$ instituição FAIPE (O Instituto de Pesquisa e Ensino - IPÊ) o protocolo de utilização do fio Bioforce inicia dependendo da severidade do apinhamento. A instituição preconiza o uso do fio Bioforce no aparelho autoligável, para o melhor aproveitamento das suas características mecânicas, porém não é uma regra, sendo que o fio Bioforce pode ser utilizado em qualquer sistema de aparelho ortodôntico.

Na fase inicial, de alinhamento e nivelamento, quando o apinhamento é severo o primeiro fio utilizado é o fio 0.014 " Niti, no entanto, quando o apinhamento é suave ou moderado o tratamento inicia com o fio Bioforce 0.019"x0.025". Para a inserção do fio em áreas com maior apinhamento é sugerido o uso de substancia refrigerante como Endo Frost $-50^{\circ}$ para ativálo e deixar assim mais maleável e facilitar sua inserção totalmente na canaleta. Para a ativação total do fio é recomendado a imersão do mesmo em água gelada pouco antes da sua inserção nos braquetes.

$\mathrm{Na}$ fase de assentamento do torque o mesmo fio Bioforce é utilizado em média por três a seis meses sem a troca do mesmo durante as manutenções, para que ocorra a expressão da prescrição do braquetes. É um fio retangular de Niti, já inserido nos primeiros ou no primeiro mês de tratamento, ou seja, desde o início o fio já preenche melhor a canaleta do braquete, tornando-o mais eficiente, o braquete já começa a expressar seus torques desde o início do tratamento. 
$\mathrm{Na}$ fase de trabalho após o alinhamento e nivelamento e o assentamento dos torques o próximo fio inserido é um fio retangular de aço 0.019 ” x 0.025 ” para execução das mecânicas mais elaboradas: fechamento de espaços de extrações, diastemas, más oclusões verticais ou horizontais.

Na fase de finalização é utilizado fio 0.018 " aço, com dobras e elásticos de intercuspidação se necessário.

Quadro 2. Protocolo de uso dos fios pela Faculdade Faipe.

\begin{tabular}{|c|c|}
\hline Protocolo Faipe (ap. autoligável Morelli) & Fios utilizados \\
\hline $1^{\text {a }}$ Fase: alinhamento e nivelamento & $0.014 " \mathrm{NiTi}$ ou Bioforce retangular 0.019”x0.025" \\
\hline $2^{\mathrm{a}}$ Fase: assentamento de torques & Bioforce retangular 0.019 "x 0.025 " por 6 meses sem troca \\
\hline $\begin{array}{l}3^{\text {a }} \text { Fase: fechamento de espaços e má-oclusões } \\
\text { verticais/verticais }\end{array}$ & $0.019 " \times 0.025$ " retangular aço \\
\hline $4^{\mathrm{a}}$ Fase: finalização & 0.018 " aço \\
\hline
\end{tabular}

Fonte: Autores.

\section{Considerações Finais}

Através desta revisão de literatura observamos que:

Para um melhor aproveitamento das características do aparelho autoligado as marcas pesquisadas neste trabalho, orientam que no mesmo, sejam utilizados fios ortodônticos tecnológicos neste aparelho (fios supereslástico ou termoativados com adição de cobre).

O fio Bioforce é considerado o primeiro fio biologicamente correto, pois permite que a força seja distribuída de forma gradual, aplicando forças baixas e suaves em dentes anteriores (100g) e aumentando em direção aos dentes posteriores (300g).

\section{Referências}

Albuquerque, C. B. et al. (2017) Deflection and Flexural Strength Effects on the Roughness of Aesthetic-Coated Orthodontic Wires. Brazilian Dental Journal, $28(1), 40-45$.

Bradley, T. G. et al. (2013) An investigation into the mechanical and aesthetic properties of new generation coated nickel-titanium wires in the as-received state and after clinical use. European Journal of Orthodontics, 36(3), 290-296.

Braga, L. C. C. et al. (2011) Friction force on brackets generated by stainless steel wire and superelastic wires with and without IonGuard. Dental Press Journal of Orthodontics, 16(4), 41.e1-41. e6.

Da Motta, A. T. S. et al. (2003) Histórico de fios utilizados em ortodontia. Ortodontia Gaúcha, 7(1), 65-70.

Gravina, M. A. et al. (2004) Fios ortodônticos: propriedades mecânicas relevantes e aplicação clínica. Revista Dental Press de Ortodontia e Ortopedia Facial, 9, 113-128.

Gurgel, J. A., Ramor, A. L., \& Kerr, S. D. (2001) Fios Ortodônticos, Revista Dental Press de Ortodontia e Ortopedia Facial, 6(4), $103-114$.

Lijima, M. et al. (2011) Effect of coating on properties of esthetic orthodontic nickel-titanium wires. Angle Orthodontist, 82(2), 319-325.

Macena, M. C. B. et al. (2015) Fios ortodônticos, propriedades microestruturais e suas aplicações clínicas: visão geral. Revista. Saúde \& Ciência Online, 4(2), 90-108.

Mandall, N. A. et al. (2006) Which orthodontic archwire sequence? A randomized clinical trial. European Journal of Orthodontics., $28(6), 561-566$.

Pereira, A. S., Shitsuka, D. M., Parreira, F. J., \& Shitsuka, R. (2018). Metodologia da pesquisa científica. https://repositorio.ufsm.br/bitstream/handle/1/15824/

Lic_Computacao_Metodologia-Pesquisa-Cientifica.pdf?sequence=1

Philip, N. et al. (2016) Newer Orthodontic Archwires: Imparting Efficacy to Esthetics. International Journal of Oral Health Dentistry, 2(2), $102-105$. 
Research, Society and Development, v. 10, n. 4, e47610414064, 2021

(CC BY 4.0) | ISSN 2525-3409 | DOI: http://dx.doi.org/10.33448/rsd-v10i4.14064

Quintão, C. C. A. \& Brunharo, I. H. V. P. (2009) Fios ortodônticos: conhecer para otimizar a aplicação clínica. Revista Dental Press de Ortodontia e Ortopedia Facial, 14(6), 144-157.

Ranjan, N. et al. (2017) Recent advancement in orthodontic wires: a review. Journal of the Dental College Azamgarh, 3(1), 50-62.

Sathler, R. et al. (2011) Desmistificando os braquetes autoligáveis. Dental Press Journal of Orthodontics, 16(2), e1-e8.

Schemann-Miguel, F. et al. (2012) Análise comparativa da relação carga/deflexão de fios retangulares de NiTi convencionais e termoativados. Dental Press Journal of Orthodontics, 17(3), 35.

Spini, T. S. et al. (2012) Avaliação do intervalo de transição térmica das ligas de níquel-titânio termoativadas. Ortodontia, 45(5), 575-580.

Ursi, W. \& Almeida, G. (2010) Ortodontia com Braquetes Autoligáveis. Almeida, M. R. Ortodontia Clínica e Biomecânica. Maringá, Ed. Dental Press, 608p., Cap. 15.

Vilella, O. V. (2007) O desenvolvimento da ortodontia no Brasil e no mundo. Dental Press Journal of Orthodontics, 12(6), 131-156.

Zanelato, R. C. T. (2016) Tratamento ortodôntico com aparelho autoligado passivo-Smart Clip ${ }^{\mathrm{TM}}$. Caso clínico https://pt.scribd.com/document/480 362833/SO78-4as-p48-54 